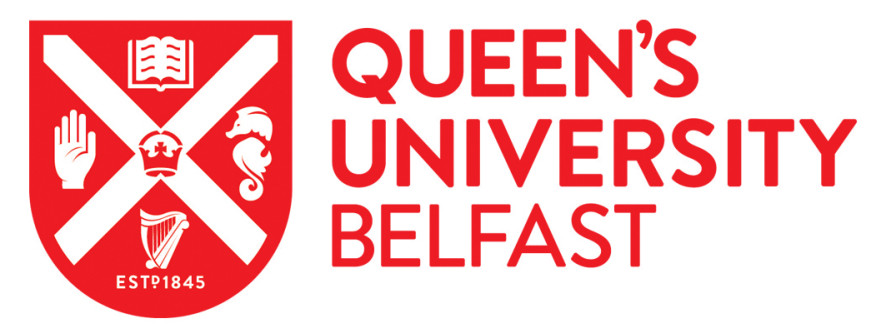

\title{
Microscopic origin of channeled flow in lamellar titanium aluminide
}

Katzarov, I., \& Paxton, A. T. (2010). Microscopic origin of channeled flow in lamellar titanium aluminide. Physical Review Letters, 104(22), 225502-1-225502-4. [225502]. https://doi.org/10.1103/PhysRevLett.104.225502

\author{
Published in: \\ Physical Review Letters
}

Document Version:

Publisher's PDF, also known as Version of record

Queen's University Belfast - Research Portal:

Link to publication record in Queen's University Belfast Research Portal

\section{Publisher rights}

Copyright 2010 The American Physical Society

This work is made available online in accordance with the publisher's policies. Please refer to any applicable terms of use of the publisher.

\section{General rights}

Copyright for the publications made accessible via the Queen's University Belfast Research Portal is retained by the author(s) and / or other copyright owners and it is a condition of accessing these publications that users recognise and abide by the legal requirements associated with these rights.

Take down policy

The Research Portal is Queen's institutional repository that provides access to Queen's research output. Every effort has been made to ensure that content in the Research Portal does not infringe any person's rights, or applicable UK laws. If you discover content in the Research Portal that you believe breaches copyright or violates any law, please contact openaccess@qub.ac.uk. 


\title{
Microscopic Origin of Channeled Flow in Lamellar Titanium Aluminide
}

\author{
Ivaylo H. Katzarov and Anthony T. Paxton \\ Atomistic Simulation Centre, School of Mathematics and Physics, Queen's University Belfast, Belfast BT7 1NN, United Kingdom
}

(Received 19 March 2010; published 2 June 2010)

\begin{abstract}
We employ a quantum mechanical bond order potential in an atomistic simulation of channeled flow. We show that the original hypothesis that this is achieved by a cooperative deployment of slip and twinning is correct, first because a twin is able to "protect" a $60^{\circ}$ ordinary dislocation from becoming sessile, and second because the two processes are found to be activated by Peierls stresses of similar magnitude. In addition we show an explicit demonstration of the lateral growth of a twin, again at a similar level of stress. Thus these simultaneous processes are shown to be capable of channeling deformation into the observed state of plane strain in so-called " $A$ "-oriented mechanical testing of titanium aluminide superalloy.
\end{abstract}

DOI: 10.1103/PhysRevLett.104.225502

In the tensile or compressive mechanical deformation of engineering alloys one expects flow to be concentrated in planes roughly at $45^{\circ}$ to the loading axis. One peculiar exception to this rule is the so called "channeled flow" in lamellar $\gamma$-TiAl superalloy. Because the atomistic understanding of plasticity and work hardening in metals remains one of the outstanding challenges in materials science today a detailed study of channeled flow is instructive. We do this using very large scale quantum mechanical simulations of dislocations under impressed strains and are able to clear up this anomaly.

Lamellar $\gamma$-TiAl is constructed of layers of crystal, upwards of $10 \mathrm{~nm}$ thick, sharing a common [111] direction and separated by grain boundaries characterized by rotations of $60^{\circ}, 120^{\circ}$ and $180^{\circ}$ about this common axis, Fig. 1(a). One might think that this structure would be more resistant to dislocation glide than the monolithic crystal since the lamellar boundaries (LB) may well be opaque or at least resistant to the transmission of slip [1,2]. On the other hand, the core structures of dislocations are strongly modified by the elastic field and "chemical presence" of lamellar boundaries such as to render otherwise sessile dislocations glissile [2]. In the so called " $A$ " orientation of the compressive axis we would expect slip to occur on, say, (111) planes that intersect the lamellar boundaries. Instead one observes no macroscopic shape change in the direction perpendicular to the LBs while the strains parallel and perpendicular to the compressive axis are equal and opposite [3]. In other words the material is in a state of constant volume plane strain. This unusual and curious phenomenon has been termed "channeled flow" [4] and has been supposed to result from the cooperative action of slip and twinning - processes usually regarded as mutually exclusive. Figure 1(b) illustrates the combination of one ordinary dislocation and three twinning dislocations on the (111) composition plane that carries a large Schmid factor in $A$ orientation and intersects the (111) lamellar boundaries on which the Schmid factor is zero. The resulting Burgers vector lies in the LBs and provides the net deformation observed in channeled flow [5].
PACS numbers: 61.72.Lk, 61.72.Bb, 61.72.Mm, 68.35.bd

We have made very large scale atomistic simulations of dislocations and lamellar boundaries under stress using an explicitly quantum mechanical prescription of interatomic forces, namely, a bond order potential. Full details have been published elsewhere [1,6,7], but we need to point out that complex intermetallics cannot be described by classical pair functionals such as embedded atom models, partly because neglected angular forces are responsible for Cauchy pressure anomalies that abound in intermetallics and partly because they cannot reliably predict planar fault energies and hence the stable core structures of dislocations. In contrast, bond order potentials are fitted to all the independent elastic constants and the potential we use for $\gamma$ TiAl is known to reproduce planar fault energies that are fully consistent with those predicted by the local density functional theory [8]. A rectangular parallelepiped simulation block consisting of 2016 active and 1056 inert atoms and one periodic unit wide along the [101] direction is used in all simulations. Shear stress, $\tau_{x z}$, is applied incrementally, in steps of $0.001 c_{44}$, to the block by applying the appropriate homogeneous shear strain, evaluated within anisotropic elasticity theory. This strain is superimposed on the dislocation displacement fields at each atomic site. Atoms are relaxed by molecular statics at every incremental step in the applied stress. We set the $x, y$, and $z$ axes of the simulation cell parallel to the [12̄1], [111], and [10ī] directions of the $L 1_{0}$ unit cell so that $\tau_{x z}$ amounts to a shear

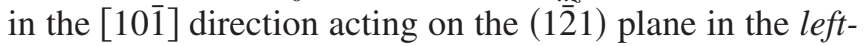
hand crystal in Fig. 2. The sense of the shear is such that it generates Peach-Koehler force on the dislocation pushing it in a direction perpendicular to the lamellar boundary.

We begin by creating a twin, three planes wide on the (111) slip plane. This is done by introducing three $\frac{1}{6}[1 \overline{1} \overline{2}]$ twinning dislocations whose elastic centers lie on the intersection of the slip plane with a $180^{\circ} \mathrm{LB}$. As shown in Fig. 2(a) this introduces a step in the boundary-incipient indication of shape change normal to the LBs which is counter to what is observed in $A$-oriented specimens. We apply a shear stress, in the plane perpendicular to the LB, and find that we can cause slip to propagate into the 

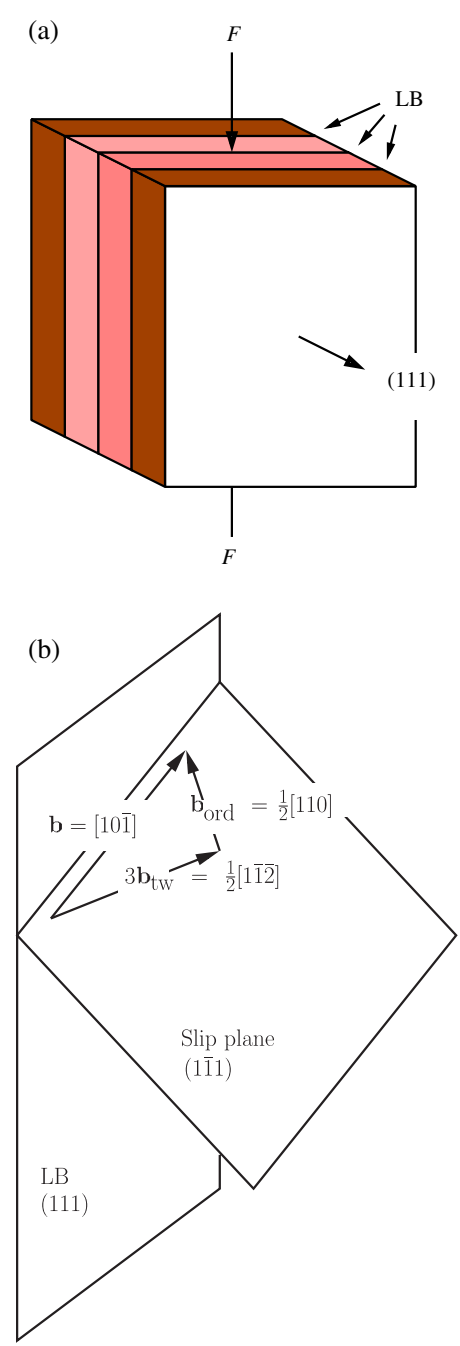

FIG. 1 (color online). (a) Schematic of the $\gamma$-TiAl lamellar microstructure loaded under compression in $A$ orientation. The domains share a common [111] direction. Orientation relations are, $60^{\circ}:[10 \overline{1}]\left\|[1 \overline{1} 0], 120^{\circ}:[10 \overline{1}]\right\|[0 \overline{1} 1], 180^{\circ}: \quad[10 \overline{1}] \|$ [101]. (b) Illustration of the total Burgers vector arising from a combination of three twinning and one ordinary $60^{\circ}$ dislocation (after Ref. [5]).

adjoining grain through the emission of a $\frac{1}{6}[1 \overline{1} \overline{2}]$ twinning dislocation. This process is illustrated in Fig. 2(b) which shows the twinning dislocation in the right-hand crystal trailing a superlattice intrinsic stacking fault (SISF). We find that the smallest stress required for slip to penetrate across the LB is 0.01 in units of the elastic shear constant, $c_{44}$, which units of stress we will use in all that follows. We find in contrast that the resistance to transmission of $120^{\circ}$ and $60^{\circ} \mathrm{LBs}$ is much greater-the $\frac{1}{6}[1 \overline{1} \overline{2}]$ twinning dislocation is nucleated as in the $180^{\circ} \mathrm{LB}$ but instead of penetrating the second grain, it glides in the LB trailing an SISF in the interface. We now increase the applied stress and find that a dipole of twinning dislocations is nucleated in an adjacent atomic plane to the surface of the existing twin (that is, on the (1111) slip plane) separated by an SISF.

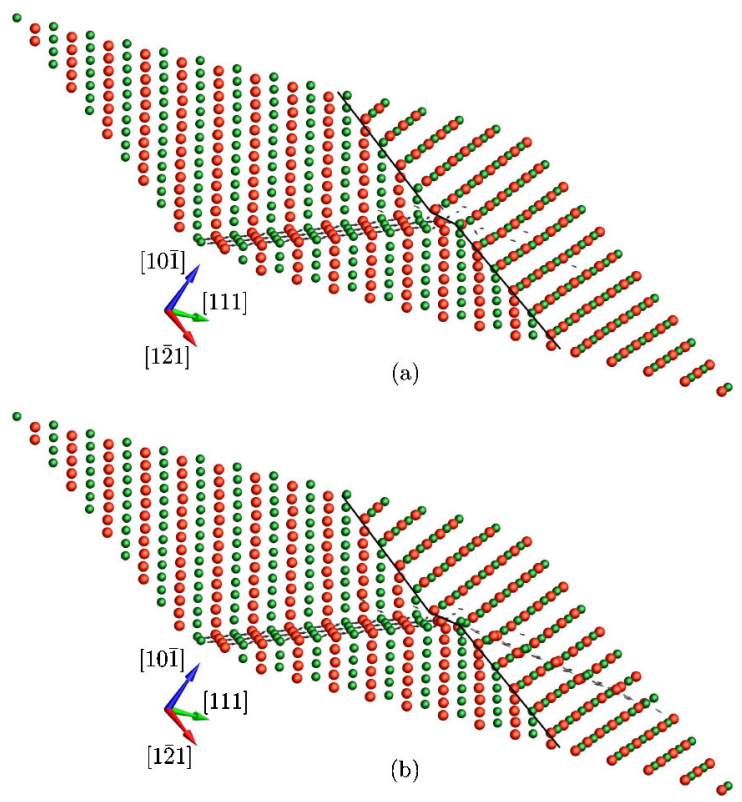

FIG. 2 (color online). (a) A three layer twin impinges upon a $180^{\circ}$ lamellar boundary. This produces a necessary step in the interface. (b) Upon application of a shear stress of $0.01 c_{44}$ perpendicular to the LB, a $\frac{1}{6}[1 \overline{1} \overline{2}]$ twinning dislocation is emitted into the adjoining grain. $\gamma \mathrm{TiAl}$ has the $L 1_{0}$ crystal structure, based on the face centered cubic lattice having alternating (002) planes populated by $\mathrm{Ti}$ and $\mathrm{Al}$ atoms, shown red and green. In this and Fig. 4, arrows show the relative edge component of displacement between the atoms on either side [15].

The expansion of the dipole at higher stresses leads to the lateral growth of the twin. The threshold stress to initiate lateral twin growth is 0.045 close to the $180^{\circ} \mathrm{LB}$ and 0.053 close to the $60^{\circ}$ and $120^{\circ} \mathrm{LBs}$. These are both smaller than the applied stress that we find necessary to prevent the collapse of the dipole in monolithic $\gamma$-TiAl in the absence of an existing twin, namely, 0.058 . Hence, we find first that lateral growth is encouraged by the presence of the existing twin and second that the nearby presence of the $180^{\circ} \mathrm{LB}$ further lowers the barrier to lateral growth. We should remark that homogeneous nucleation as a means of lateral growth is ruled out in the conventional wisdom in favor of the familiar "barber's pole" and other schemes. However, our results are consistent with a recent finding [9].

Before we come to our simulations of channeled flow, we anticipate that the step in the lamellar boundary due to the introduction of the twin in Fig. 2(a) will be eliminated by the additional Burgers vector of a $\frac{1}{2}[110]$ dislocation [see Fig. 1(b)]. Having the required [101] line sense puts this dislocation into $60^{\circ}$ mixed orientation and its behavior in monolithic $\gamma$-TiAl turns out to be qualitatively different from that of the screw dislocation that we have described earlier [10]. This is shown schematically in Fig. 3 which illustrates the following findings of our simulations. Under zero stress, the dislocation has a non planar core which extends largely on the (111) slip plane but which also spreads onto the (111) cross slip plane. When a shear stress 

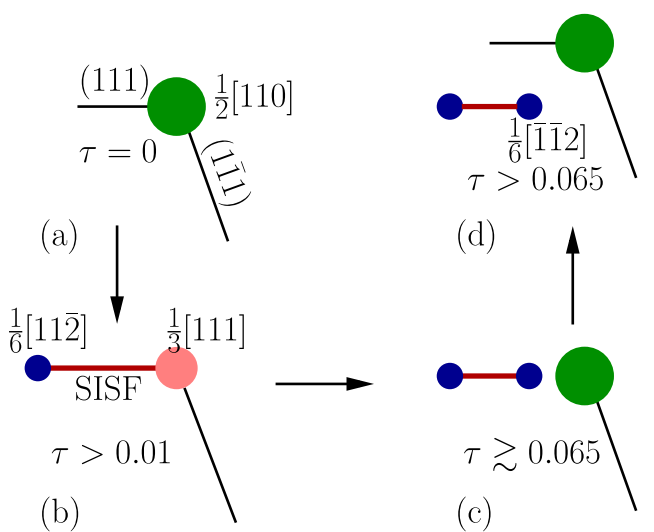

(d)

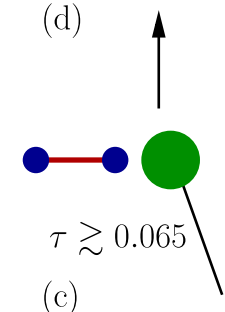

FIG. 3 (color online). A schematic of glide of the $60^{\circ}$ dislocation in monolithic $\gamma$ TiAl. (a) Under zero stress the dislocation has a nonplanar core, spread onto the slip and cross slip planes. (b) At a resolved shear stress of just $0.01 c_{44}$ the dislocation dissociates according to Eq. (1). (c) If the stress is increased, the decomposition of Eq. (2) happens resulting in recovery of the original dislocation having emitted a faulted Shockley dipole. In fact, the process is more complex than this, see the text. (d) The ordinary dislocation now glides on the slip plane leaving behind the dipole. As the stress is relieved by glide, one expects the sequence (b)-(d) to repeat resulting in a jerky flow, as observed, leaving a debris of dipoles which are expected to annihilate.

greater than 0.01 is applied, the core extends onto the cross slip plane and there is a core transformation,

$$
\frac{1}{2}[110] \rightarrow \frac{1}{6}[11 \overline{2}]+\mathrm{SISF}+\frac{1}{3}[111]
$$

such that a Shockley partial $\frac{1}{6}[11 \overline{2}]$ dislocation is emitted onto the cross slip plane trailing SISF and leaving a sessile $\frac{1}{3}[111]$ Frank partial at the original elastic center. If the stress is increased to 0.065 , then two consecutive events happen: First a dipole of Shockley partials is nucleated by the stress to the right of the Frank partial in Fig. 3. Second, the left-hand dislocation in this dipole combines with the Frank partial to recreate the original $60^{\circ}$ dislocation which glides upward. The remaining right-hand partial glides towards the first emitted Shockley to create the dipole illustrated in Fig. 3(c). This complex event amounts to a further transformation of the Frank partial in which it appears to emit another twinning partial with opposite sense to the first:

$$
\frac{1}{3}[111] \rightarrow \frac{1}{6}\left[\begin{array}{lll}
\overline{1} & \overline{1} & 2
\end{array}\right]+\frac{1}{2}[110] .
$$

The dislocation now glides on its slip plane and given that the stress is relieved slightly, it may stop and repeat the process, resulting in a rather jerky progress.

We now create a three layer microtwin, as in Fig. 2(a), terminating at a $180^{\circ}$ lamellar boundary and we place a $60^{\circ}$ ordinary dislocation within the twin but with its elastic center some distance from the LB. The relaxed structure shown in Fig. 4(a) indicates that the step is eliminated at the intersection of the twin with the LB. Now we apply a glide force on (111) in such a sense as to drive the ordinary dislocation towards the LB. Two things happen; see Fig. 4(b). First, the dislocation does not cross slip, or dissociate on the (111) cross slip plane; instead it glides on (111) at a Peierls stress of 0.04 . The second thing to happen is the lateral growth of the twin by nucleation of a dipole on an adjacent (111) plane as described earlier.

It is interesting to note two further observations. (i) We have created our three layer microtwin in monolithic $\gamma$-TiAl and we find that the three partial $\frac{1}{6}[1 \overline{1} \overline{2}]$ dislocations glide in parallel at a Peierls stress of 0.01 . Hence the longitudinal growth of the twin may occur at very low

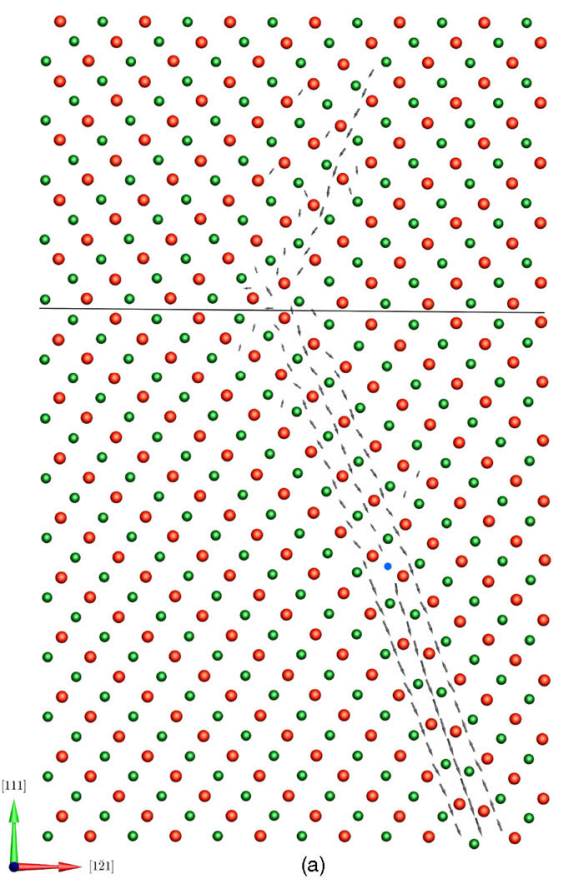

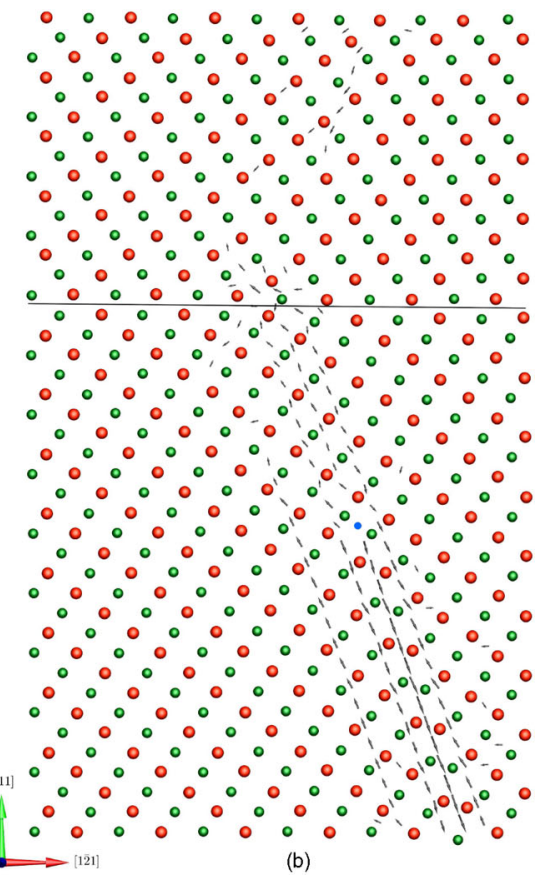

225502-3
FIG. 4 (color online). A $60^{\circ} \frac{1}{2}[110]$ dislocation is placed within the twin with elastic center indicated by a blue dot. (b) Under shear stress on the (1111) composition plane of the twin the $60^{\circ}$ dislocation glides towards the LB; simultaneously a $\frac{1}{6}[1 \overline{1} \overline{2}]$ twinning partial dipole is nucleated which expands under stress producing an SISF parallel to the twin boundary. 
stress. (ii) We have caused this growing twin to impinge upon our $60^{\circ}$ dislocation and find that the latter does not become incorporated into the twin; instead it acts as an obstacle to further twin advance. It therefore appears essential to the cooperative deployment of slip and twinning that is the signature of channeled flow that the $60^{\circ}$ dislocation is introduced into an existing twin.

In summary, molecular statics simulations at zero Kelvin have revealed the following. (i) The interfacial step at a LB necessarily introduced by the impingement of three $\frac{1}{6}[1 \overline{1} \overline{2}]$ twinning dislocations is canceled by a single $\frac{1}{2}[110] 60^{\circ}$ dislocation, whose elastic center is near but not necessarily at the LB. (ii) Dipole nucleation and lateral twin growth is initiated at the same Peierls stress, $\sim 0.04$, as the motion of ordinary dislocations. (iii) The Peierls stress for longitudinal growth of twins is 0.01 - significantly lower than that for ordinary dislocations. (iv) A $60^{\circ}$ dislocation in monolithic $\gamma$-TiAl has non planar core and a Peierls stress of 0.065. At stresses close to this it emits Shockley partial dipoles as it glides. In contrast, the same dislocation with elastic center inside a microtwin has a non planar core, but does not dissociate according to Eq. (1), and has much lower Peierls stress of $\sim 0.04$. We have confirmed this for three and six layer twins. (v) $120^{\circ}$ and $60^{\circ}$ lamellar boundaries act as obstacles to transmission of both twins and dislocations. Once a twin has impinged upon a LB, further stress causes the twin to grow laterally while longitudinal growth is impeded by the LB. (6) The cooperative action of slip and twinning serves to translate a resolved shear on the (111) slip plane into a plane strain deformation with invariant plane parallel to the (111) LB plane. This is consistent with macroscopic observation and confirms earlier speculations [3,5].

By a comparison of calculated Peierls stresses we have demonstrated that slip and twinning are able to combine on similar length and time scales. In real life, deformation happens at finite temperature and we normally expect ordinary dislocation velocities to be dominated by kink pair nucleation as is usual for dislocations with non planar cores; in the special case of the $60^{\circ}$ ordinary dislocations gliding within and on the composition plane of a microtwin we expect glide to be dominated by kink pair formation to a very much lesser extent. The formation of twins is expected to occur at the lamellar boundaries following the emission of twinning dislocations [2,11]. We have now shown that lateral growth of twins may be accomplished at the same levels of stress as the propagation of dislocations. This is probably the first explicit demonstration of spontaneous nucleation of a dipole pair leading to the widening of a twin. We cannot be sure how ordinary dislocations are nucleated inside microtwins, but we expect this to happen at the lamellar boundaries by the reaction of twinning partials with interfacial dislocations [12]. There is evidently a natural association of twins and dislocations but we do not always expect the cancelation of Fig. 1(b) to be accomplished perfectly. If the twinning and ordinary dislocations do not occur in the exact ratio of three to one, then some strain normal to the lamellar boundaries on the scale of some hundred nanometers is to be expected; this is consistent with experiment [13].

The picture that emerges in monolithic $\gamma$-TiAl is of a $60^{\circ}$ dislocation moving between self-locking events at each of which it emits a Shockley partial dipole. This jerky flow is observed [14], but the atomistic mechanism that we advance here is a new one. We do not expect the emitted dipoles to be observable since once the stress is removed they will annihilate. The key to the operation of channeled flow is the observation that the $60^{\circ}$ dislocation is protected by its surrounding twin from the dissociation of Eq. (1) and consequent self-locking, and becomes glissile. It may then conspire with its containing microtwin to channel the flow into a plane strain condition.

We are grateful to Vaclav Vitek for valuable conversations and to Dimitar Pashov for help with the figures. This work was supported by the UK EPSRC under grant No. EP/E025854/1.

[1] I. H. Katzarov, M. J. Cawkwell, A. T. Paxton, and M. W. Finnis, Philos. Mag. 87, 1795 (2007).

[2] I. H. Katzarov and A. T. Paxton, Acta Mater. 57, 3349 (2009).

[3] K. Kishida, H. Inui, and M. Yamaguchi, Philos. Mag. A 78, 1 (1998).

[4] M.-C. Kim, M. Nomura, V. Vitek, and D.P. Pope, Materials Research Society Symposium Proceedings, Vol. 552, p. KK3.1.1 (MRS, Pittsburgh, PA, 1999).

[5] Y. Chen, D. P. Pope, and V. Vitek, Materials Research Society Symposium Proceedings, Vol. 753, p. BB3.10.1 (MRS, Pittsburgh, PA, 2003).

[6] A. P. Horsfield, A. M. Bratkovsky, M. Fearn, D. G. Pettifor, and M. Aoki, Phys. Rev. B 53, 12694 (1996).

[7] M. J. Cawkwell, D. Nguyen-Manh, C. Woodward, D. G. Pettifor, and V. Vitek, Science 309, 1059 (2005).

[8] S. Znam, D. Nguyen-Manh, D. G. Pettifor, and V. Vitek, Philos. Mag. A 83, 415 (2003).

[9] J. Li, K. J. V. Vliet, T. Zhu, S. Yip, and S. Suresh, Nature (London) 418, 307 (2002).

[10] I. H. Katzarov and A. T. Paxton, Philos. Mag. 89, 1731 (2009).

[11] Twinning in Advanced Materials, edited by M. H. Yoo and M. Wuttig (TMS, Warrendale, PA, 1994).

[12] F. Appel and R. Wagner, Materials Science \& Engineering 22, 187 (1998).

[13] Y. Chen and D.P. Pope, Materials Research Society Symposium Proceedings, Vol. 842, p. S6.5.1 (MRS, Pittsburgh, PA, 2005).

[14] A. Couret, Intermetallics 9, 899 (2001).

[15] See supplementary material at http://link.aps.org/supplemental/10.1103/PhysRevLett.104.225502 to see quantitative detail in enlarged views of Figs. 2 and 4. Additional displacement maps and further details may be obtained by application to the authors. 